\title{
Energy gap reduction in dilute nitride GaAsSbN
}

\author{
Yan-Ting Lin, Ta-Chun Ma, Tsung-Yi Chen, and Hao-Hsiung Lin ${ }^{\text {a) }}$ \\ Graduate Institute of Electronics Engineering and Department of Electrical Engineering, \\ National Taiwan University, Taipei 10617, Taiwan
}

(Received 13 August 2008; accepted 7 October 2008; published online 30 October 2008)

\begin{abstract}
The energy gap of dilute nitride GaAsSbN has been studied. We found that the energy gap reduction induced by nitrogen incorporation is nearly independent of the $\mathrm{Sb}$ composition of the alloy, indicating that the conduction band and the valence band can be independently manipulated by incorporating $\mathrm{N}$ and $\mathrm{Sb}$, respectively. A "double" band anticrossing (BAC) model, which is a combination of a BAC model for GaAsN and a valence BAC model for GaAsSb with the localized levels and hybridization parameters reported in literatures, has been proposed to fit the energy gap of annealed GaAsSbN samples. The as-grown samples, however, are with lower energy gaps, most likely resulting from the existence of substitutional N pairing and clustering in the alloys. (C) 2008 American Institute of Physics. [DOI: 10.1063/1.3009199]
\end{abstract}

$\mathrm{GaAsSbN}$ is a promising material for GaAs-based long wavelength applications because the alloy can be grown exactly lattice-matched to GaAs with an energy gap much narrower than GaAs. ${ }^{1}$ Energy gap as low as $0.8 \mathrm{eV}$ has been reported recently, ${ }^{2}$ which indicates the feasibility of applications to GaAs-based or Ge-based solar cells and photodetectors working for fiber communications. ${ }^{3,4}$ The composition dependency of the band structure and the band alignment in GaAsSbN alloys are essential for theoretical modeling and device design. Two-level band anticrossing (BAC) model has demonstrated its capability of explaining the energy gap reduction in dilute nitrides. ${ }^{5,6}$ Moreover, the model also predicts several important effects observed in dilute nitrides including the enhancement of the electron effective mass, ${ }^{7}$ the increment in the donor activation efficiency in $\mathrm{InGaAsN},{ }^{8}$ and the transition from indirect to direct energy gap in GaPN. ${ }^{9}$ However, there are very few reports discussing the BAC model for GaAsSbN. ${ }^{10}$ As compared with InGaAs, GaAsSb has much smaller conduction band offset and stronger energy gap bowing. Though the band alignment of GaAs/ $\mathrm{GaSb}$ system is not very certain yet, the reported conduction band offset of the system has been within a quite small range, from $+0.12 \mathrm{eV}$ (type-II lineup) to $-0.2 \mathrm{eV}$ (type-I lineup). ${ }^{11-13}$ This small offset leads the conduction band minimum of As-rich GaAsSb almost independent of the $\mathrm{Sb}$ composition, which provides GaAsSbN a nearly invariant host band for BAC model. The large bowing parameter of GaAsSb reveals that BAC model is applicable to this ternary alloy. Wu et al. ${ }^{6}$ calculated the conduction band structure of Sb-rich GaAsSb using BAC model. In a recent paper, Alberi et al. ${ }^{14}$ showed a valence BAC (VBAC) model that is capable of explaining the energy gap As-rich GaAsSb. In this paper, we use a double BAC model, a combination of a conduction band BAC model and a VBAC model to calculate the band structure of GaAsSbN. The results are in good agreement with the experimental energy gaps determined from absorption measurement.

Figure 1 is a schematic diagram showing two BAC models, which are used to calculate the energies of the conduction band, valence band, and spin-orbit split valence band of

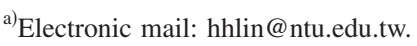

$\mathrm{GaAs}_{1-x-y} \mathrm{Sb}_{x} \mathrm{~N}_{y}$. On the top of the figure is a model modified from the BAC model for GaAsN reported in literatures. ${ }^{6}$ The localized level of $\mathrm{N}$ is $0.23 \mathrm{eV}$ above the conduction band minimum of GaAs. According to the BAC model, the conduction band is split into upper and lower subbands whose energies are given as follows:

$$
E_{ \pm}^{C}=\frac{E_{\mathrm{CB}}+E_{\mathrm{N}} \pm \sqrt{\left(E_{\mathrm{CB}}-E_{\mathrm{N}}\right)^{2}+4 V_{\mathrm{NCB}}^{2}}}{2},
$$

where $E_{\mathrm{N}}$ is the energy of the $\mathrm{N}$ state, $E_{\mathrm{CB}}$ is the conduction band minimum of the $\mathrm{N}$-free GaAsSb matrix, and $V_{\mathrm{NCB}}$ is the matrix element coupling those two types of states. $V_{\mathrm{NCB}}$ $=C_{\mathrm{N}} \mathrm{y}^{1 / 2}$, where $C_{\mathrm{N}}$ is the hybridization parameter and $y$ is the mole fraction of $\mathrm{N}$. Here, virtual crystal approximation (VCA) is used to describe the conduction band minimum of $\mathrm{GaAsSb}$, and the formula is

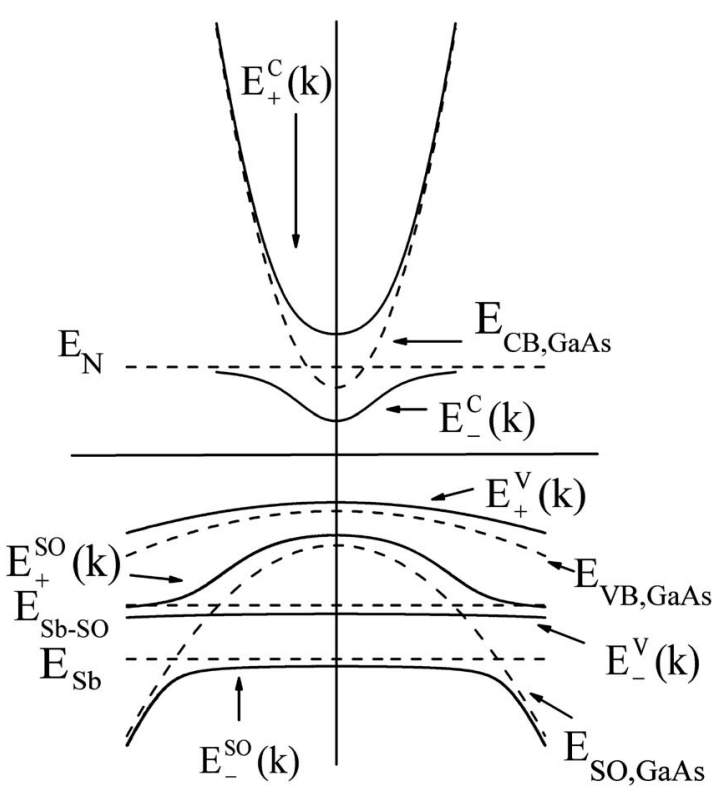

FIG. 1. Schematic diagram showing the two BAC models, which are used to calculate the energies of the conduction band, valence band, and spin-orbit split band of $\mathrm{GaAs}_{1-x-y} \mathrm{Sb}_{x} \mathrm{~N}_{y}$. 
(a)

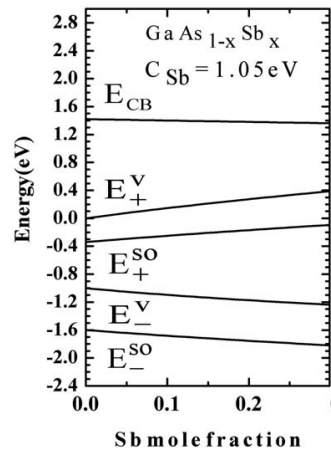

(b)

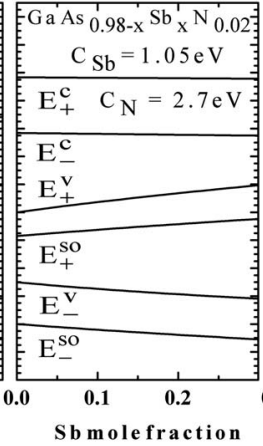

(c)

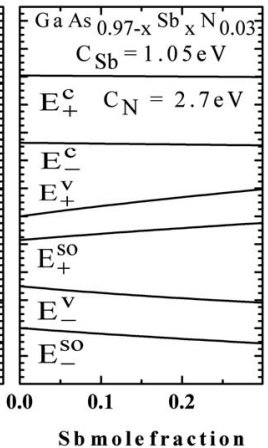

FIG. 2. Calculated band structures for (a) GaAsSb, (b) $\mathrm{GaAs}_{0.98-x} \mathrm{Sb}_{x} \mathrm{~N}_{0.02}$, and (c) $\mathrm{GaAs}_{0.97-x} \mathrm{Sb}_{x} \mathrm{~N}_{0.03}$.

$$
E_{\mathrm{CB}}=E_{\mathrm{CB}, \mathrm{GaAs}}-\Delta E_{\mathrm{CBM}} x,
$$

where $E_{\mathrm{CB}, \mathrm{GaAs}}$ is the conduction band of GaAs, $\Delta E_{\mathrm{CBM}}$ is the conduction band offset between the two end-point compounds of GaAsSb, i.e., GaAs and GaSb, and $x$ is the mole fraction of $\mathrm{Sb}$. The band offset adopted in this work is $0.2 \mathrm{eV}^{11}$

On the bottom of Fig. 1 is a VBAC model for GaAsSb. Alberi et al. $^{14}$ developed a VBAC model with the realm of the $\mathbf{k} \cdot \mathbf{p}$ formalism. They derived a $12 \times 12$ matrix Hamiltonian for the six valence bands and the relevant $\mathrm{Sb}$ levels. For simplicity, we consider the $\Gamma$ point only. Since $k=0$ in this case, the $12 \times 12$ matrix can be reduced to six $2 \times 2$ matrices with the form of the two-level model. The split levels for the tree bands at $k=0$ have the form similar to Eq. (1) and can be expressed as follows:

$$
E_{ \pm}^{V}=\frac{E_{\mathrm{VB}}+E_{\mathrm{Sb}} \pm \sqrt{\left(E_{\mathrm{VB}}-E_{\mathrm{Sb}}\right)^{2}+4 V_{\mathrm{SbVB}}^{2}}}{2}
$$

where $E_{\mathrm{Sb}}$ is the energy level of the atomic Sb states corresponding to the valence bands, $E_{\mathrm{VB}}$ is the energy level at $k$ $=0$ for the three valence bands of the host matrix, and $V_{\mathrm{SbVB}}$ is the matrix element coupling the $\mathrm{Sb}$ level and the valence band. $V_{\mathrm{SbVB}}=C_{\mathrm{Sb}} x^{1 / 2}$, where $C_{\mathrm{Sb}}$ is the hybridization parameter. The heavy and light hole bands are degenerate at $k=0$. Their corresponding $p$-like $\mathrm{Sb}$ level is $1.0 \mathrm{eV}$ below the valence band maximum (VBM) of GaAs and the hybridization parameter is $1.05 \mathrm{eV}^{14}$ For spin-orbit band, the corresponding level is the atomic spin-orbit splitting energy of $\mathrm{Sb}$, which is located at $1.6 \mathrm{eV}$ below the VBM of GaAs. ${ }^{14,15}$ The hybridization energy for spin-orbit band is also $1.05 \mathrm{eV}$. Again, VCA is used to describe the valence bands with the formula

$$
E_{\mathrm{VB}}=E_{\mathrm{VB}, \mathrm{GaAs}}-\Delta E_{\mathrm{VBM}} x,
$$

where $E_{\mathrm{VB}, \mathrm{GaAs}}$ is the valence bands at $k=0, \Delta E_{\mathrm{VBM}}$ is the band offset between GaAs and GaSb. The band offsets $\Delta E_{\mathrm{VBM}}$ of the degenerated heavy and light hole bands and the spin-orbit band are $0.5 \mathrm{eV}$, and $0.09 \mathrm{eV}$, respectively. ${ }^{14}$ The calculated results for GaAsSb, $\mathrm{GaAs}_{0.98-x} \mathrm{Sb}_{x} \mathrm{~N}_{0.02}$, and $\mathrm{GaAs}_{0.97-x} \mathrm{Sb}_{x} \mathrm{~N}_{0.03}$ are shown in Fig. 2. As can be seen, $E_{+}^{C}$ and $E_{-}^{C}$ are virtually independent of the Sb mole fraction. The slight declines are due to the small type-I band offset adopted in this study.

Two series of GaAsSbN with $2 \%$ and $3 \% \mathrm{~N}$ composition were prepared by gas source molecular epitaxy. Detailed

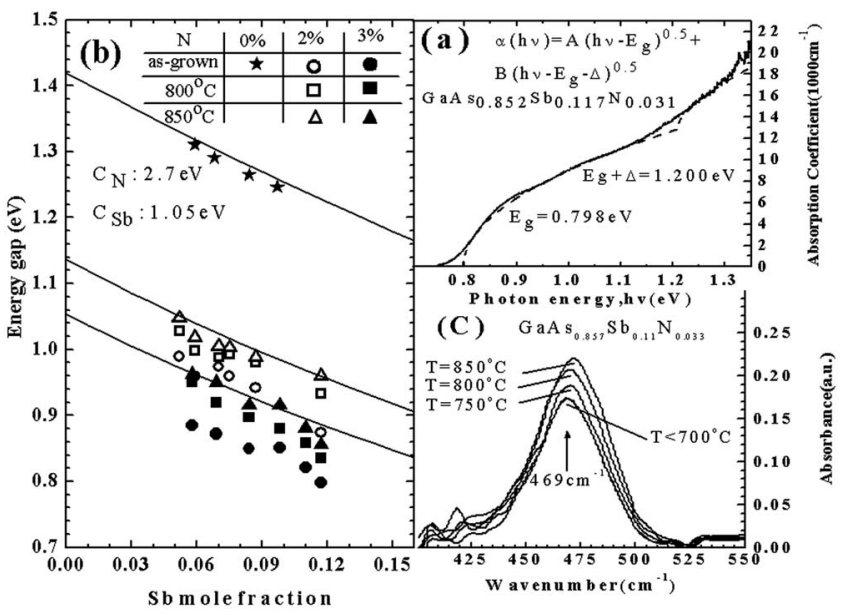

FIG. 3. (a) Absorption spectrum of an as-grown GaAsSbN sample. The fitting curve, considering both the $E_{V}^{+}$to $E_{C}^{-}$and $E_{S O}^{+}$to $E_{C}^{-}$transitions, is also depicted in the figure. The energy gap and spin-orbit splitting energy of the GaAsSbN sample are determined from curve fitting. (b) Energy gaps of the as-grown and annealed GaAsSbN samples with $2 \%$ and $3 \% \mathrm{~N}$ mole fraction as functions of $\mathrm{Sb}$ mole fraction. Solid curves are the simulation results with different $\mathrm{N}$ mole fractions, $0 \%, 2 \%$, and $3 \%$. (c) $\mathrm{Ga}-\mathrm{N}$ vibration mode absorption spectra of a typical GaAsSbN sample before and after therma annealing.

growth procedure has been published elsewhere. ${ }^{2}$ The lattice mismatches of these samples are within $\pm 6 \times 10^{-3}$. Because all the samples are $1 \mu \mathrm{m}$ in thickness, the strain effect on the energy gap is negligible. The compositions were determined by a JEOL JAX-8200 electron probe microanalysis system. To characterize the absorption spectra, an ASB-W030 tungsten-halogen lamp was used as the light source. The light was dispersed by an SPEX 500M monochromator and was collected by an InGaAs photodiode using standard lock-in technique. After the growth, the samples were annealed for $5 \mathrm{~min}$ at 800 and $850{ }^{\circ} \mathrm{C}$ by a rapid thermally annealer in $\mathrm{N}_{2}$ ambience.

The absorption spectrum of a typical as-grown GaAsSbN sample is shown in Fig. 3(a). The energy gap $E_{g}$ and spin-orbit splitting energy $\Delta$ of the sample are obtained by fitting the absorption coefficient $\alpha$ using the following formula: 16

$$
\begin{aligned}
\alpha(h \nu) & =A \sqrt{h \nu-E_{g}} \text { for } E_{g}+\Delta \geq h \nu \geq E_{g}, \\
& =A \sqrt{h \nu-E_{g}}+B \sqrt{h \nu-\left(E_{g}+\Delta\right)} \text { for } h \nu \geq E_{g}+\Delta,
\end{aligned}
$$

where $h$ is Planck's constant, $\nu$ is the photon frequency, and $A$ and $B$ are constants. As can be seen, the energy gap and the splitting energy of the $\mathrm{GaAs}_{0.872} \mathrm{Sb}_{0.098} \mathrm{~N}_{0.03}$ sample are 0.798 and $0.402 \mathrm{eV}$, respectively. Note that the energy gap is quite close to that of Ge. However, strong below band edge absorption is also observed. Compositional fluctuation in diluted nitride material ${ }^{17}$ is the origin of the strong absorption below energy gap. Figure 3(b) shows the measured band gaps of the as-grown and annealed GaAsSbN samples with $2 \%$ and $3 \% \mathrm{~N}$ mole fraction as functions of Sb mole fraction. Three curves representing the energy gaps of GaAsSb, $\mathrm{GaAs}_{0.98-x} \mathrm{Sb}_{x} \mathrm{~N}_{0.02}$, and $\mathrm{GaAs}_{0.97-x} \mathrm{Sb}_{x} \mathrm{~N}_{0.03}$, calculated by the combination BAC model, are also plotted in the figure. In order to cross check the validity of GaAsSb curve, we intentionally grew four GaAsSb samples. The results, as shown in the figure, are in good agreement with the curve. The energy 


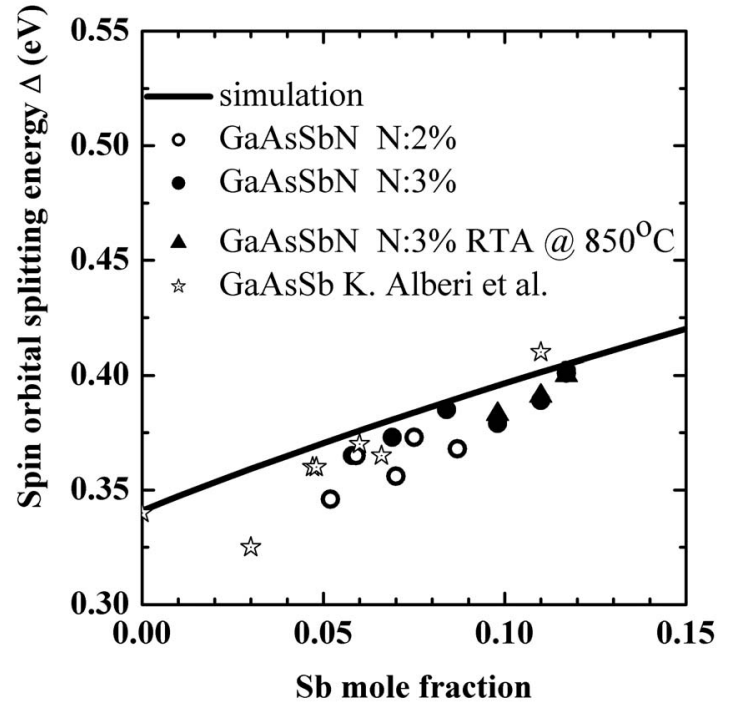

FIG. 4. Spin-orbit splitting energies of GaAsSbN samples as a function of $\mathrm{Sb}$ mole fraction. For comparison, the splitting energies of GaAsSb alloys reported by K. Alberi et al. (Ref. 14) are also plotted.

gaps of the as-grown GaAsSbN samples are below the calculated curve. However, after the thermal annealing, the energy gaps blueshift and approach their corresponding curves. When the annealing temperature reaches $850{ }^{\circ} \mathrm{C}$, the energy gaps well fit the curves. Note that the parameters of the conduction BAC (CBAC) model, including the hybridization parameter of $2.7 \mathrm{eV}$, are taken from Ref. 6, which have been verified for GaAsN. The deviation of the as-grown samples most likely results from the existence of substitutional $\mathrm{N}$ pairing or clustering in the samples. Bellaiche and Zunger ${ }^{18}$ predicted that such short range orders should induce a redshift with respect to the random alloy. Ciatto et al. ${ }^{19}$ suggested that thermal treatment tends to break these pairing and clustering and randomize the nitrogen atoms. Consequently, the energy gaps blueshift and fit the corresponding curves. In fact, we have measured the Ga-N vibration mode of these samples using Fourier transform infrared spectroscopy. A typical result is shown in the inset of Fig. 3(c). The absorbance of the $\mathrm{Ga}-\mathrm{N}$ mode increases as the energy gap blueshifts revealing the change in atoms in the group- $\mathrm{V}$ sublattice due to the thermal annealing.

Note that the two GaAsSbN curves in Fig. 3(b) are parallel to the GaAsSb curve, indicating that the energy gap reduction due to $\mathrm{N}$ incorporation is independent of $\mathrm{Sb}$ mole fraction. Since the N-induced reduction results from the interaction between the $\mathrm{N}$ level and the conduction band of $\mathrm{GaAsSb}$, this finding implies that the conduction band energy of GaAsSb is virtually invariant of $\mathrm{Sb}$ composition; otherwise the reduction in energy gap would be a function of $\mathrm{Sb}$ composition. In fact, the studies on the band alignment of GaAs/GaAsSb support this point. ${ }^{11-13}$ The change in energy gap in GaAsSb is mostly in the valence band. Our finding also suggests that the conduction band energy and the valence band energy of GaAsSbN can be independently manipulated by adding $\mathrm{N}$ and $\mathrm{Sb}$ into the alloy, respectively, which is important for band gap engineering.
Figure 4 shows the spin-orbit energies of the GaAsSbN samples as a function of $\mathrm{Sb}$ mole fraction. As can be seen, the results are fairly close to the calculated curve. It is worth noting that there is almost no difference between the asgrown and annealed samples, indicating the blueshift after thermal annealing is indeed relevant to the $\mathrm{N}$ atoms. In addition, the results of GaAsSb reported in Ref. 14 are also depicted for comparison and in good agreement with the results of GaAsSbN samples because the incorporation of $\mathrm{N}$ only affects the conduction bands.

In conclusion, we have studied the energy gap of dilute nitride GaAsSbN. The energy gap reduction resulting from nitrogen incorporation is independent of the $\mathrm{Sb}$ mole fraction, indicating that the conduction band and the valence band are independently controlled by incorporating $\mathrm{N}$ and $\mathrm{Sb}$ in, respectively. A double BAC model, consisting of a CBAC model and a VBAC model is proposed and explains the energy gap of GaAsSbN.

This work was supported by the National Science Council of R.O.C. and Institute of Nuclear Energy Research, Atomic Energy Council of R.O.C. under Contract No. NSC96-NU-7-002-003.

${ }^{1}$ J. C. Harmand, A. Caliman, E. V. K. Rao, L. Largeau, J. Ramos, R. Teissier, L. Travers, G. Ungaro, B. Theys, and I. F. L. Dias, Semicond. Sci. Technol. 17, 778 (2002).

${ }^{2}$ T. C. Ma, Y. T. Lin, and H. H. Lin, J. Cryst. Growth 310, 2854 (2008).

${ }^{3}$ D. B. Jackrel, S. R. Bank, H. B. Yuen, M. A. Wistey, J. S. Harris, A. J. Ptak, S. W. Johnston, D. J. Friedman, and S. R. Kurtz, J. Appl. Phys. 101, 114916 (2007).

${ }^{4}$ S. Wicaksono, S. F. Yoon, W. K. Loke, K. H. Tan, and B. K. Ng, J. Appl. Phys. 99, 104502 (2006).

${ }^{5}$ W. Shan, W. Walukiewicz, J. W. Ager III, E. E. Haller, J. F. Geisz, D. J. Friedman, J. M. Olson, and S. R. Kurtz, Phys. Rev. Lett. 82, 1221 (1999).

${ }^{6} \mathrm{~J}$. Wu, W. Shan, and W. Walukiewicz, Semicond. Sci. Technol. 17, 860 (2002).

${ }^{7}$ C. Skierbiszewski, P. Perlin, P. Wisniewski, W. Knap, T. Suski, W. Walukeiwica, W. Shan, K. M. Yu, J. W. Ager, E. E. Haller, J. F. Geisz, and J. M. Olson, Appl. Phys. Lett. 76, 2409 (2000).

${ }^{8}$ K. M. Yu, W. Walukiewicz, W. Shan, J. W. Ager III, J. Wu, E. E. Haller, J. F. Geisz, D. J. Friedman, and J. M. Olson, Phys. Rev. B 61, R13337 (2000)

${ }^{9}$ W. Shan, W. Walukiewicz, K. M. Yu, J. Wu, J. W. Ager III, E. E. Haller, H. P. Xin, and C. W. Tu, Appl. Phys. Lett. 76, 3251 (2000).

${ }^{10}$ Y. X. Dang, W. J. Fan, S. T. Ng, S. Wicaksono, S. F. Yoon, and D. H. Zhang, J. Appl. Phys. 98, 026102 (2005).

${ }^{11}$ S. Tiwari and D. J. Frank, Appl. Phys. Lett. 60, 630 (1992).

${ }^{12}$ G. Liu, S. L. Chuang, and S. H. Park, J. Appl. Phys. 88, 5554 (2000).

${ }^{13}$ J. B. Wang, S. R. Johnson, S. A. Chaparro, D. Ding, Y. Cao, Yu. G. Sadofyev, Y. H. Zhang, J. A. Gupta, and C. Z. Guo, Phys. Rev. B 70, 195339 (2004).

${ }^{14}$ K. Alberi, J. Wu, W. Walukiewicz, K. M. Yu, O. D. Dubon, S. P. Watkins, C. X. Wang, X. Liu, Y. J. Cho, and J. Furdyna, Phys. Rev. B 75, 045203 (2007).

${ }^{15}$ X. Gonze, J. P. Michenaud, and J. P. Vigneron, Phys. Rev. B 41, 11827 (1990).

${ }^{16} \mathrm{~V}$. Swaminathan and A. T. Macrander, Materials Aspects of GaAs and InP Based Structures (Prentice-Hall, New Jersey, 1991), p. 267.

${ }^{17}$ E. H. Reihlen, M. J. Jou, Z. M. Fang, and G. B. Stringfellow, J. Appl. Phys. 68, 4604 (1990).

${ }^{18}$ L. Bellaiche and A. Zunger, Phys. Rev. B 57, 4425 (1998).

${ }^{19}$ C. Ciatto, J.-C. Harmand, F. Glas, L. Largeau, M. Le Du, F. Boscherini, M. Malvestuto, L. Floreano, P. Glatzel, and R. Alonso Mori, Phys. Rev. B 75, 245212 (2007). 